UCRHEP-T510

September 2011

\title{
Hiding the Higgs Boson from Prying Eyes
}

\author{
Ernest Ma \\ Department of Physics and Astronomy, University of California, \\ Riverside, California 92521, USA
}

\begin{abstract}
There are two simple ways that the Higgs boson $H$ of the Standard Model (SM) may be more difficult to observe than expected at the Large Hadron Collider (LHC) or the Tevatron. One is well-known, i.e. $H$ decays invisibly, into dark-matter scalar particles for example. The other is that $H$ mixes with a heavy singlet scalar $S$ which couples to new colored fermions and scalars. Of the two mass eigenstates, the light one could (accidentally) have a supppressed effective coupling to two gluons, and the heavy one could be kinematically beyond the reach of the LHC.
\end{abstract}


The one Higgs boson [1] $H$ of the Standard Model (SM) of particle interactions is expected to be produced by gluon-gluon fusion at the Large Hadron Collider (LHC) and be observed through its decay into $Z Z, W W$, and other channels. Recent reported data [2, 3, 4] have excluded the following mass ranges at $95 \%$ confidence level:

$$
\begin{aligned}
\text { ATLAS : } & 146-232,256-282, \quad 296-466 \mathrm{GeV}, \\
\text { CMS : } & 145-216,226-288,310-340 \mathrm{GeV}, \\
\text { TEVATRON : } & 156-177 \mathrm{GeV} .
\end{aligned}
$$

Combined with the LEPII bound [5] of $m_{H}>114.4 \mathrm{GeV}$, this leaves only a small window for its observation. Whereas more data could eventually find $H$ through its rare decay mode to two photons, it is perhaps a good time now to consider how $H$ may be hidden from view because of either its decay or its production.

A first possibility is that $H$ decays significantly into invisible channels, thereby diminishing its branching fractions into observable final states. This is a very old idea [6] and has many different model realizations. One recent example is the model of a dark (inert) scalar doublet [7], where the Standard Model is extended to include a second scalar doublet, which is odd under an exactly conserved $Z_{2}$ symmetry [8]. If the neutral member $\eta^{0}=\left(\eta_{R}+i \eta_{I}\right) / \sqrt{2}$ of this doublet is split so that $m_{R}<m_{I}$ by at least the order of $100 \mathrm{keV}$, then $\eta_{R}$ is a good dark-matter candidate [9, 10]. For the latest discussion on this model, see Ref. [11]. If $2 m_{R}<m_{H}$, then the invisible decay of $H$ into these dark-matter scalars will suppress its branching fractions to other particles, as already discussed in detail a few years ago [12]. The effect is especially significant below the $W W$ threshold and could suppress the $\gamma \gamma$ branching fraction by as much as a factor of three.

A second possibility is a new proposal. The idea is very simple. Suppose there is a scalar singlet $S$ which couples to new colored fermions and scalars. In that case, both $H$ and $S$ will couple to two gluons through loops. Let the $H g g$ amplitude be $A_{H}$ and the $S g g$ amplitude be 
$A_{S}$, then $A_{H}$ is dominated by the $t$-quark loop, and $A_{S}$ comes from the new colored fermions and scalars. Take for example $A_{S}=3 A_{H}$. Now if $H$ mixes with $S$, the linear combination $H^{\prime}=(3 H-S) / \sqrt{10}$ would not couple to two gluons, and would not be produced at the LHC by gluon-gluon fusion. If $H^{\prime}$ also happens to be a mass eigenstate, then it could hide from being seen at the LHC even if its mass is $170 \mathrm{GeV}$ (above the $W W$ threshold). The orthogonal combination $S^{\prime}=(3 S+H) / \sqrt{10}$ has an enhanced coupling to two gluons, but it is presumably heavy because it is mostly a singlet, and could be kinematically beyond the reach of the LHC.

Consider the scalar potential of the SM doublet $\Phi=\left(\phi^{+}, \phi^{0}\right)$ and a real singlet $S$ :

$$
V=\mu_{1}^{2} \Phi^{\dagger} \Phi+\frac{1}{2} \lambda_{1}\left(\Phi^{\dagger} \Phi\right)^{2}+\frac{1}{2} \mu_{2}^{2} S^{2}+\frac{1}{3} \mu_{3} S^{3}+\frac{1}{4} \lambda_{2} S^{4}+\mu_{4} S \Phi^{\dagger} \Phi+\frac{1}{2} \lambda_{3} S^{2} \Phi^{\dagger} \Phi .
$$

Let $\left\langle\phi^{0}\right\rangle=v$ and $\langle S\rangle=u$, then the minimum of $V$ is determined by

$$
\begin{aligned}
& 0=v\left(2 \mu_{1}^{2}+2 \lambda_{1} v^{2}+\lambda_{3} u^{2}+2 \mu_{4} u\right), \\
& 0=u\left(\mu_{2}^{2}+\lambda_{2} u^{2}+\lambda_{3} v^{2}+\mu_{3} u\right)+\mu_{4} v^{2} .
\end{aligned}
$$

The $2 \times 2$ mass-squared matrix spanning the physical scalars $H$ and $S$ is given by

$$
\mathcal{M}^{2}=\left(\begin{array}{cc}
2 \lambda_{1} v^{2} & \sqrt{2}\left(\lambda_{3} u+\mu_{4}\right) v \\
\sqrt{2}\left(\lambda_{3} u+\mu_{4}\right) v & 2 \lambda_{2} u^{2}+\mu_{3} u-\mu_{4} v^{2} / u
\end{array}\right) .
$$

Let the mass eigenstates of the above be $H^{\prime}=H \cos \theta-S \sin \theta$ and $S^{\prime}=S \cos \theta+H \sin \theta$, with eigenvalues $m_{1}^{2}$ and $m_{2}^{2}$, then Eq. (7) may be rewritten as

$$
\mathcal{M}^{2}=\left(\begin{array}{ll}
m_{1}^{2} \cos ^{2} \theta+m_{2}^{2} \sin ^{2} \theta & \left(m_{2}^{2}-m_{1}^{2}\right) \sin \theta \cos \theta \\
\left(m_{2}^{2}-m_{1}^{2}\right) \sin \theta \cos \theta & m_{1}^{2} \sin ^{2} \theta+m_{2}^{2} \cos ^{2} \theta
\end{array}\right) .
$$

As an example, let $\sin \theta=1 / \sqrt{10}, \cos \theta=3 / \sqrt{10}, u=2 \sqrt{2} v=492.4 \mathrm{GeV}$, where $v=174.1$ $\mathrm{GeV}$, we then obtain $m_{1}=170 \mathrm{GeV}$ and $m_{2}=500 \mathrm{GeV}$ for the choice $\lambda_{1}=0.84, \lambda_{2}=0.47$, $\lambda_{3}=0.55$, and $\mu_{3}=\mu_{4}=0$. This demonstrates the numerical viability of this proposal.

It has been assumed that $S$ couples to new colored fermions and scalars. This is of course model-dependent, but a necessary condition is to have $A_{S}=A(S \rightarrow g g)$ a few times larger 
than $A_{H}=A(H \rightarrow g g)$. Now $A_{H}$ is dominated by the $t$ quark which is a fundamental triplet under $S U(3)_{C}$ and is proportional to $(\sqrt{2} v)^{-1}$. Suppose $A_{S}$ comes from a colored fermion octet $Q$ with the coupling $S \bar{Q} Q$, then it is proportional to $u^{-1}$ but its color factor of 3 is 6 times that of the $t$ quark. Hence for the above choice of $u=2 \sqrt{2} v, A_{S} \simeq 3 A_{H}$ is realized. The allowed mass term $\bar{Q} Q$ would change the details of the above, but may be forbidden by a $Z_{2}$ symmetry under which $S$ and $Q_{L}$ are odd, but $Q_{R}$ is even.

More realistically, $H^{\prime}$ is unlikely to decouple from $g g$ entirely. In that case, the suppression (or enhancement if $\sin \theta<0$ ) factor in $H^{\prime}$ production at the LHC is $\left(\cos \theta-\left(A_{S} / A_{H}\right) \sin \theta\right)^{2}$. On the other hand, depending on the choice of new colored fermions and scalars, there is also a contribution from $A(S \rightarrow \gamma \gamma)$ to $H^{\prime}$ decay. This means that the branching fraction of $H^{\prime}$ to $\gamma \gamma$ would also not be the same as in the SM. If a particle is discovered at the LHC in the $\gamma \gamma$ channel below $145 \mathrm{GeV}$, but with a branching fraction different from what is expected from the SM, especially if it is greater, it may be due to this effect. The presence of the octet $Q$ may also be relevant in gauge-coupling unfication [13] without supersymmetry.

In conclusion, the existence of the Higgs boson may be hidden from view at present because of a variety of scenarios, some of which have been discussed recently [14, 15, 16, 17]. In this paper, two simple ways are considered: the presence of light dark-matter scalars which affects the decay or an accidental cancellation between $A(H \rightarrow g g)$ and $A(S \rightarrow g g)$ in $H-S$ mixing which affects the production. In the latter case, an increase from the present $E_{c m}=7 \mathrm{TeV}$ to $14 \mathrm{TeV}$ at the LHC in the future would produce $S^{\prime}$ easily, and the decay $S^{\prime} \rightarrow H^{\prime} H^{\prime}$ would be a spectacular signature for discovering $H^{\prime}$.

I thank Bohdan Grzadkowski and Maria Krawczyk for a stimulating "Scalars 2011" Conference in Warsaw (August 2011) which led to this work. My research is supported in part by the U. S. Department of Energy under Grant No. DE-AC02-06CH11357. 


\section{References}

[1] For a review, see for example A. Djouadi, Phys. Rep. 457, 1 (2008).

[2] A. Nisati, Talk at the XXV International Symposium on Lepton Photon Interactions at High Energies (Mumbai, India), August 2011.

[3] V. Sharma, Talk at the XXV International Symposium on Lepton Photon Interactions at High Energies (Mumbai, India), August 2011.

[4] M. Verzocchi, Talk at the XXV International Symposium on Lepton Photon Interactions at High Energies (Mumbai, India), August 2011.

[5] Particle Data Group: K. Nakamura et al., J. Phys. G: Nucl. Part. Phys. 37, 075021 (2010).

[6] R. E. Shrock and M. Suzuki, Phys. Lett. B110, 250 (1982).

[7] E. Ma, Phys. Rev. D73, 077301 (2006).

[8] N. G. Deshpande and E. Ma, Phys. Rev. D18, 2574 (1978).

[9] R. Barbieri, L. J. Hall, and V. S. Rychkov, Phys. Rev. D74, 015007 (2006).

[10] L. Lopez Honorez, E. Nezri, J. F. Oliver, and M. H. G. Tytgat, JCAP 0702, 028 (2007).

[11] L. Lopez Honorez and C. E. Yaguna, JCAP 1101, 002 (2011).

[12] Q.-H. Cao, E. Ma, and G. Rajasekaran, Phys. Rev. D76, 095011 (2007).

[13] E. Ma, Phys. Lett. B625, 76 (2005).

[14] F. Bonnet, M. B. Gavela, T. Ota, and W. Winter, arXiv:1105.5140 [hep-ph].

[15] K. Ghosh, B. Mukhopadhyaya, and U. Sarkar, Phys. Rev. D84, 015017 (2011) arXiv:1105.5837 [hep-ph]].

[16] X.-G. He and G. Valencia, arXiv:1108.0222 [hep-ph].

[17] X.-G. He and J. Tandean, arXiv:1109.1277 [hep-ph]. 